\title{
Oligomeric alkylpyridinium surfactants prepared via ATRP
}

\author{
Xin Su, ${ }^{2}$ Zonglin Chu, ${ }^{12}$ Ya Shuai, ${ }^{12}$ Zanru Guo, ${ }^{12}$ Yujun Feng ${ }^{1 *}$ \\ $1^{*}$ Chengdu Institute of Organic Chemistry, Chinese Academy of Sciences, Chengdu \\ 610041, Sichuan, P. R. China; tel.: +86-28-85236874; fax: +86-28-85236874; e-mail: \\ yjfeng@cioc.ac.cn. \\ 'Graduate University of the Chinese Academy of Sciences, Beijing 100039, P. R. \\ China.
}

(Received: 03 December, 2009; published: 11 May, 2011 )

\begin{abstract}
A series of oligomeric alkylpyridinium surfactants were prepared directly in aqueous media by atom transfer radical polymerization (ATRP) of a surfmer, 4vinyl-N-dodecylpyridinium bromide, and their surface activities were examined in comparison with the surfmer. The resulting oligomers have narrow molecular weight distributions, with polydispersity indices in the range of 1.17-1.23. By using 2-morpholinoethyl 2-bromo-2-methylpropanoate as ATRP initiator, the molecular weight of oligomeric surfactants was characterized by ${ }^{1} \mathrm{H}$ NMR and the results were close to those obtained from GPC analysis. It was found that critical micelle concentration (CMC) of the oligomeric alkylpyridinium surfactants shifted to lower concentrations with increasing degree of polymerization $\left(D P_{n}\right)$, and their $\gamma_{\mathrm{cmc}}$ values were smaller by about 4 to $8 \mathrm{mN} / \mathrm{m}$ than that of the corresponding surfmer. Among the series of surfactants, the oligomer with highest $\mathrm{DP}_{\mathrm{n}}$ showed the greatest efficiency in lowering the surface tension in aqueous solution.
\end{abstract}

\section{Introduction}

Oligomeric surfactants are a novel family of amphiphilic compounds made up of two or more conventional surfactant "monomers" linked by spacer groups at the level of, or very close to the head groups [1]. Scientifically, these oligomers could bridge the low-molecular-weight traditional surfactants and high-molecular-weight polymers; for the industries, much interest has been generated by the fact that oligomeric surfactants show great enhancements in many of the properties typically shown by surfactants [1-3]. Compared with their single-tail monomeric counterparts, oligomeric surfactants are more efficient in reducing the surface tension of water, and have much lower critical micelle concentration (CMC), higher surface activity, stronger viscosifying ability and unusual self-assembly [1-6]. Consequently, they show many potential applications such as effective emulsifiers, dispersants, foaming agents, detergents, etc. [1].

Though they have received increasing scientific and commercial attention, it is hard to synthesize oligomeric surfactants via traditional organic processes. Two typical routes were reported so far to prepare oligomeric cationic surfactants. The first one is one-step method [4-6] in which N,N,N,N",N"-pentamethyl diethylenetriamine was directly quaternized with bromoalkane to get trimeric cationic surfactants. Such a reaction is readily operated and the obtained raw products could be easily purified; however, tedious long time (more than ten days!) is needed and very low yield $(\sim 10 \%)$ is obtained [5]. The other is multi-step process [3,7-9] in which methylamine was used as starting material via four steps to synthesize trimeric quaternary ammonium 
surfactant [3]; but complicated procedures and difficult synthesis are inevitable drawbacks of such a process. Although improved performances were evidenced for oligomeric surfactants $[1,10-14]$, only a limited number of trimeric and tetrameric surfactants appeared to date [2-9], owing to the impediment in the synthesis.

To overcome the limitations of the above organic processes, we attempt to prepare oligomeric surfactants by shifting our efforts toward to the polymerization of surfmers, i.e., polymerizable surfactants that combine the physical behaviours of surfactants with the chemical reactivity of monomers $[15,16]$. Though surfmers have been widely employed to make microporous materials $[17,18]$, organized hydrogels and nanolatex particles $[19,20]$ by traditional free-radical polymerization, it is unlikely that this method could be sufficient to produce oligomers with controlled structure. Therefore, it is desirable to develop a suitable procedure that could polymerize surfmers into controlled oligomers, and atom transfer radical polymerization (ATRP) $[21,22]$ is one of the choices to meet such a goal. The beauty of this technique lies in precise control over degree of polymerization $\left(\mathrm{DP}_{\mathrm{n}}\right)$, and production of polymers with a narrow molecular-weight distribution. Furthermore, it was reported that the theoretical $\mathrm{DP}_{\mathrm{n}}$ of the polymers synthesized via ATRP could be small enough by utilizing high concentrations of initiator [23, 24].

As far as we are aware, there have been few reports on the synthesis of the oligomeric cationic surfactants via ATRP. In this paper, ATRP was used to prepare a series of oligomeric cationic surfactants from alkylpyridinium surfmer, 4-vinyl-Ndodecylpyridinium bromide (VPB12), and surface activities of the oligomers were examined in comparison with VPB12.

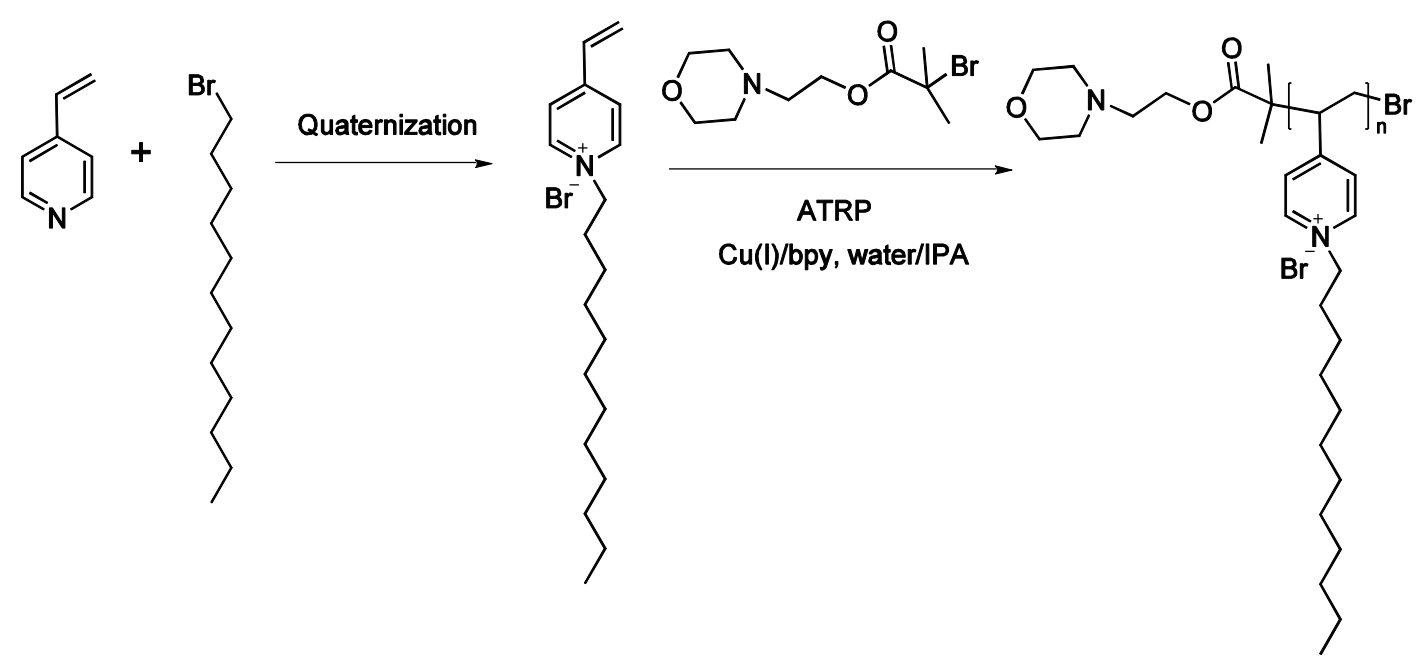

Scheme 1. The route for synthesizing oligomeric alkylpyridinium surfactants

\section{Results and discussion}

\section{Synthesis Procedure of Oligomeric Alkylpyridinium Surfactants}

The oligomeric alkylpyridinium surfactants were prepared via ATRP by a two-step process shown in Scheme 1. The preliminary step of ATRP is preparation of VPB12 surfmer by quaternization of 4-vinylpyridine with 1-bromododecane. The surfmer was almost synthesized at room temperature due to the quaternization of tertiary amine with bromoalkane with relatively low yield. It was noted that the vinylalkylpyridinium 
surfmer undergoes spontaneous polymerization even without photolytic or deliberate chemical initiation [16,17], and vinylalkylpyridinium salts are always base- or nucleophile-initiated [18].

The second step is to homopolymerize VPB12 with ATRP process. The resulting homo-oligomers are named as "PVPB12-n" where $n$ denotes the designed $D P_{n}$. Vinylalkylpyridinium polymers have been studied to some extent [25], but are generally produced by quaternization of pre-synthesized polyvinylpyridine other than polymerization of the vinylalkylpyridinium monomer. To the best of our knowledge, the vinyldodecylpyridinium oligomeric surfactants studied here are rarely mentioned in the literature, and the oligomeric surfactants synthesized via ATRP in particular have not yet been reported.

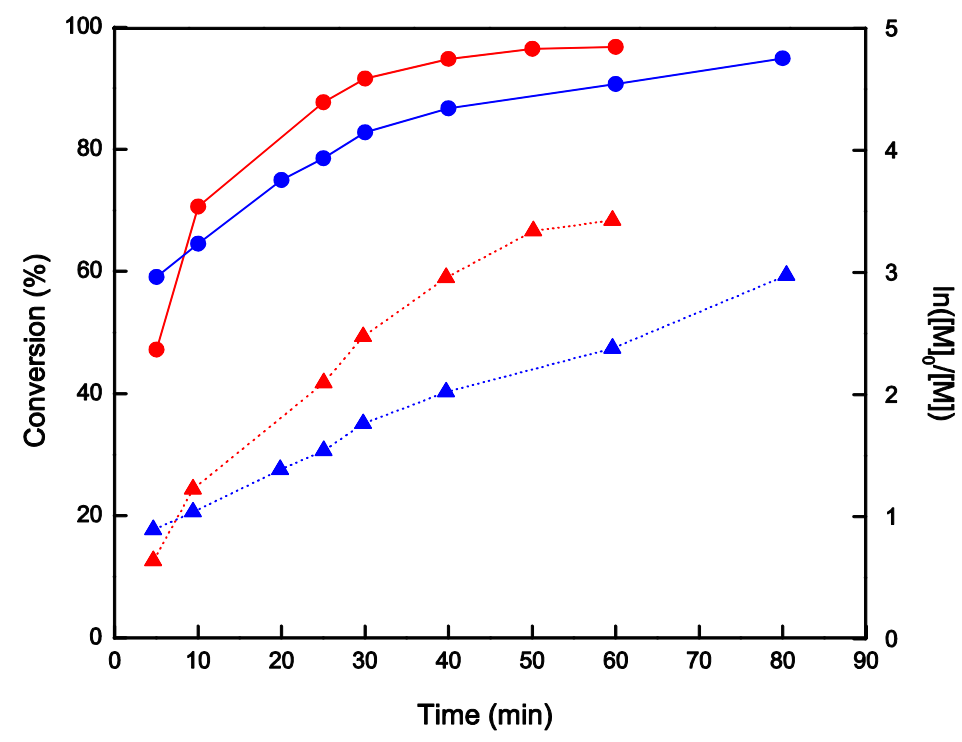

Fig. 1. Time-conversion (circles) and first-order kinetic plots (triangles) for the oligomers PVPB12-15 (red) and PVPB12-30 (blue) synthesized via ATRP.

Fig. 1 shows the first-order kinetic plots and the relationship of conversion versus reaction time for the polymerization of VBP12 in the presence of ME-Br initiator and $\mathrm{Cu}(\mathrm{I})$ complex catalyst in mixed solution at $20^{\circ} \mathrm{C}$.

Linear variation of $\ln \left([\mathrm{M}]_{0} /[\mathrm{M}]\right)$ with time, i.e., the pseudo-first-order kinetic plot, is consistent with a "living" polymerization mechanism over the first 40 min, indicating that the concentration of active propagating radical species remains almost unchanged during the early stage of oligomerization. Within the range of reaction time studied (Fig. 1), conversion of surfmer increases upon reaction time, but pronounced curvature was observed for the conversion-time curves, implying that the polymer radical concentration did not remain constant during the whole polymerization. Matyjaszewski et al. [26] reported similar results and found fast polymerization when they conducted ATRP of neutral and ionic monomers in aqueous homogeneous media. The reason of fast polymerization in aqueous ATRP is that only a small fraction of the deactivating $\mathrm{Cu}$ (II) halide complex contains a 
coordinated halide ion. Most of the $\mathrm{Cu}$ (II) complexes are halide-free and cannot deactivate radicals efficiently.

The differences in kinetic behaviour of oligomerization with different theoretical $\mathrm{DP}_{\mathrm{n}}$ was also observed, for the synthesis process of PVPB12-15, about $90 \%$ conversion was achieved after only 32 min of polymerization, while it took more than one hour to get the same conversion when PVPB12-30 was prepared. This resulted from different ratios of initiator to surfmer when synthesising oligomers with different designed $\mathrm{DP}_{\mathrm{n}}$.

Tab. 1 shows the conversions, polydispersity indices and molecular weights measured by ${ }^{1} \mathrm{H}$ NMR of $\mathrm{PVDP}_{12}-15$ and $\mathrm{PVDP}_{12}-30$. The polydispersity indices almost did not change as the polymerization proceeded. Molecular weight values increased linearly until high conversion. The theoretical molecular weight nearly coincides with the measured ones. These results indicate that the polymerization of surfmers proceeded with maintaining a living nature at least except for the final with high conversion.

Tab. 1. Results of obtained $\mathrm{PVDP}_{12}-15, \mathrm{PVDP}_{12}-30$ under different polymerization time.

\begin{tabular}{cccccc}
\hline $\mathrm{N}\left(\mathrm{VPB}_{12}\right): \mathrm{n}(\mathrm{ME}-\mathrm{Br})$ & $\begin{array}{c}\text { Reaction } \\
\text { Time }(\min )\end{array}$ & $\begin{array}{c}\text { Conversion } \\
(\%)\end{array}$ & $\begin{array}{c}\text { Theoretical } \\
\mathrm{M}_{\mathrm{n}}\end{array}$ & $\begin{array}{c}{ }^{1} \mathrm{H} \text { NMR } \\
\mathrm{M}_{\mathrm{n}}\end{array}$ & $\begin{array}{c}\text { Polydispersity } \\
\text { Indices }\end{array}$ \\
\hline $15: 1$ & 10 & 45.4 & 2592 & 2605 & 1.24 \\
& 15 & 75.1 & 4287 & 4311 & 1.23 \\
& 30 & 88.4 & 5046 & 5128 & 1.25 \\
& 35 & 90.5 & 5167 & 5203 & 1.26 \\
& 45 & 94.2 & 5378 & 5431 & 1.25 \\
& 50 & 96.4 & 5503 & 5678 & 1.25 \\
& 60 & 98.7 & 5635 & 6023 & 1.23 \\
\hline $30: 1$ & 10 & 59.4 & 6887 & 6941 & 1.21 \\
& 15 & 64.5 & 7479 & 7582 & 1.21 \\
& 25 & 74.6 & 8649 & 8736 & 1.20 \\
& 30 & 78.3 & 9078 & 9213 & 1.18 \\
& 35 & 82.5 & 9565 & 9871 & 1.21 \\
& 45 & 85.1 & 9864 & 10024 & 1.23 \\
& 60 & 90.4 & 10482 & 10683 & 1.21 \\
& 80 & 94.1 & 10911 & 11021 & 1.17 \\
\hline
\end{tabular}

Plotted in Fig. 2 is a typical example of the evolution of molecular weight determined by Gel Permeation Chromatography (GPC). Because all the GPC elution profiles are unimodal and the molecular weight distributions are relatively narrow, oligomerizations of VPB12 proceed generally in a controlled manner. PVPB12-30 has a narrowest molecular weight distribution $\left(M_{w} / M_{n}=1.17\right)$ and PVPB12-15 yielded via ATRP with a relatively broad one (Fig. 2). There is an apparent phenomenon that polydispersity index (PDI) of oligomers decreases with increasing the designed $\mathrm{DP}_{\mathrm{n}}$, because of differences in the efficiency of $\mathrm{ME}-\mathrm{Br}$ initiator to mediate the synthesis of oligomeric surfactants in aqueous media. Steady growth of theoretical $\mathrm{DP}_{\mathrm{n}}$ reduces the deviation extent between observed and theoretical molecular weight (Tab. 1). However, the $M_{n}$ values determined by GPC can only be considered as apparent 
values mainly because PEG, an uncharged polymer, was used as GPC calibration standards.

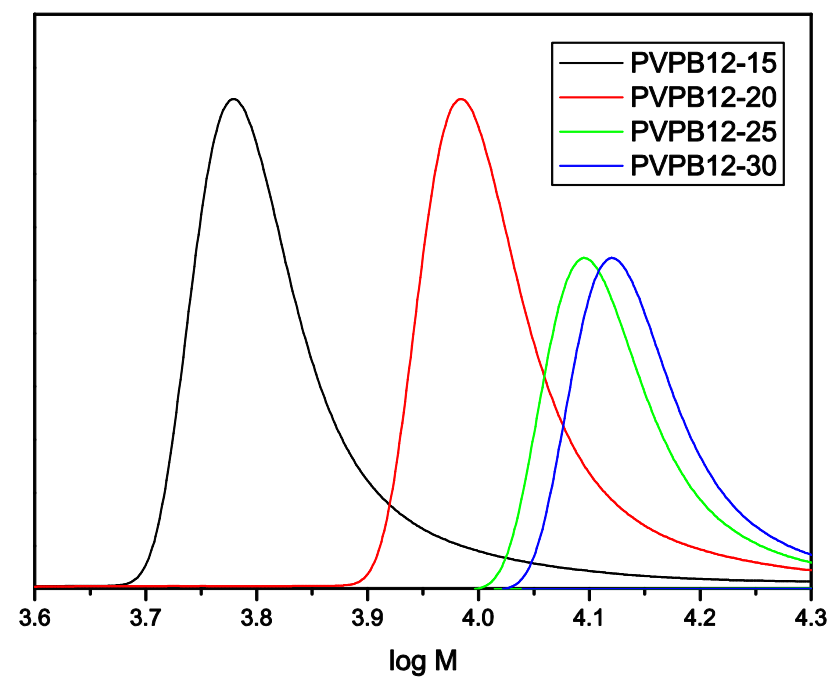

Fig. 2. GPC results for the series of oligomeric alkylpyridinium surfactants synthesized via ATRP.

Tab. 2. Molecular weight, polydispersity of the series of oligomeric alkylpyridinium surfactants.

\begin{tabular}{cccccccc}
\hline Sample & $\begin{array}{c}\text { Theor } \\
\mathrm{M}_{\mathrm{n}}\end{array}$ & $\begin{array}{c}1 \mathrm{H} \text { NMR } \\
\mathrm{M}_{\mathrm{n}}{ }^{\mathrm{a}}\end{array}$ & $\begin{array}{c}\mathrm{GPC} \\
\mathrm{M}_{\mathrm{w}}{ }^{\mathrm{b}}\end{array}$ & $\begin{array}{c}\mathrm{GPC} \\
\mathrm{M}_{\mathrm{n}}{ }^{\mathrm{b}}\end{array}$ & $\mathrm{M}_{\mathrm{w}} / \mathrm{M}_{\mathrm{n}}{ }^{\mathrm{b}}$ & $\begin{array}{c}\text { Reaction } \\
\text { Time }(\min )\end{array}$ & $\begin{array}{c}\text { Conversion } \\
(\%)\end{array}$ \\
\hline PVPB12-15 & 5635 & 6023 & 7210 & 5862 & 1.23 & 60 & 95.0 \\
PVPB12-20 & 7367 & 8015 & 10342 & 8547 & 1.21 & 80 & 94.6 \\
PVPB12-25 & 9139 & 10095 & 13079 & 11084 & 1.18 & 100 & 95.3 \\
PVPB12-30 & 10911 & 11021 & 14458 & 12357 & 1.17 & 120 & 96.4 \\
\hline
\end{tabular}

${ }^{a}$ determined by ${ }^{1} \mathrm{H}$ NMR spectroscopy. ${ }^{\circ}$ determined by gel permeation chromatography, calibrated with PEG as standards with a mobile phase of water.

${ }^{1} \mathrm{H}$ NMR spectroscopy is a useful tool to give molecular weight of polymers [27]. For example, the molecular weight of the PVPB12-n polymers can be calculated from their ${ }^{1} \mathrm{H}$ NMR spectra based on

$M_{n(N M R)}=\frac{I_{1.13} / 3}{I_{2.07} / 4} \times M_{\text {surfmer }}+280$

where $I_{1.13}$ and $I_{2.07}$ stand for the integral intensity of peaks of protons attached to methyl units in the end of branch chain and methylene units in the 4-(2hydroxyethyl)morpholine groups at the end of polymer backbone, and the " $\mathrm{M}_{\text {surfmer" }}$ and "280" refer to molecular weight of the surfmer and ME-Br, respectively. Surely, it is assumed that the initiator is consumed completely and there is no transfer in ATRP.

As compared in Tab. 1, $M_{n(N M R)}$ of oligomeric surfactant is higher than $M_{n \text { (theo) }}$ based on the assumption that all the initiator participated in the initiation of polymerization: 


$$
M_{n \text { (theo) }}=\frac{[M]_{0}}{[I]_{0}} \times M_{\text {surfmer }}+280
$$

where $[\mathrm{M}]_{0} /[]_{0}$ represents the molar ratio of the monomer and initiator in the feed composition [28]. The observed molecular weight for the oligomers is consistent with the theoretical one, which means ${ }^{1} \mathrm{H}$ NMR spectroscopy is a convenient and efficient tool for measurement of molecular weight of oligomers.

\section{Static Surface Tension}

All the four oligomeric alkylpyridinium surfactants PVPB12- $n(n=15,20,25$ and 30) have good water solubilities at ambient environment, and so their surface tension could be readily measured at $25^{\circ} \mathrm{C}$. Fig. 3 shows the static surface tension varies as a function of concentration for the series of oligomers and corresponding surfmer VPB12. The surface tension decreased with increasing surfactant concentrations and then reached clear break points, which were taken as CMC of the surfactants. The surface tension at $\mathrm{CMC}, \gamma_{\mathrm{cmc}}$, is the effectiveness of surface tension reduction.

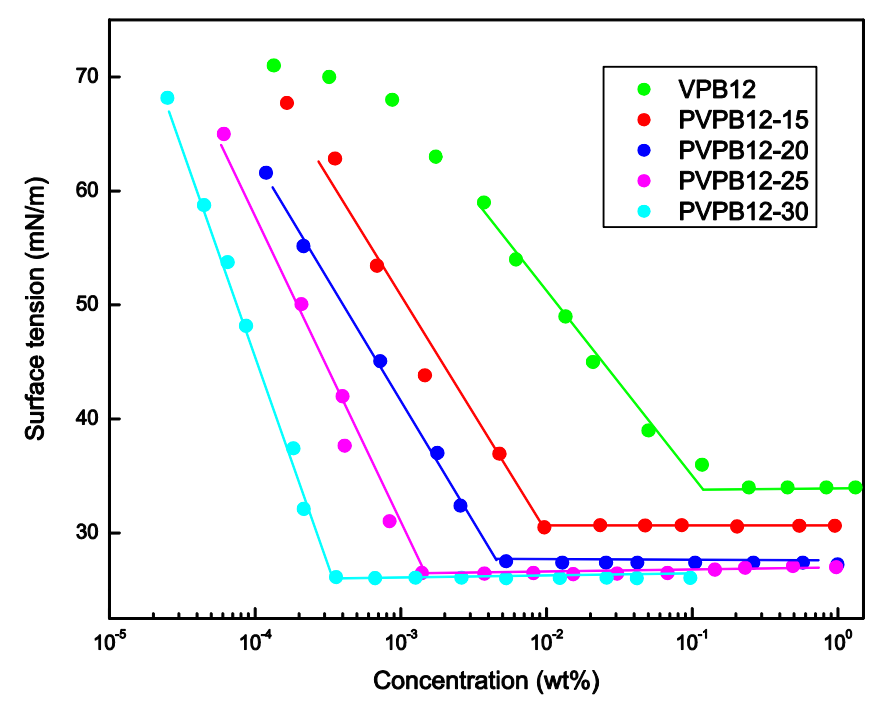

Fig. 3. Surface tension plotted as a function of concentration for aqueous solutions of the series of oligomeric surfactants and corresponding surfmer.

From Figs. 3, 4 and Tab. 3, one can easily find the general trend that both CMC and $\gamma_{C M C}$ decrease upon increasing polymerization degree. For example, the CMC of PVPB12-30 is only $1 / 3,1 / 17,1 / 30$ and $1 / 800$ of those of PVPB12-25, PVPB12-20, PVPG12-15 and corresponding monomer VPB12 that could be regarded as monomeric surfactant with polymerization degree of 1 . This means that PVPB12-30 has a much better micelle-forming ability than its surfmer and the other oligomeric surfactants. It was reported [4] that the surface activity properties of the oligomeric cationic surfactants prepared via traditional organic synthesis also follows the same rule. For instance, CMC of monomeric surfactant DTAB, gemini surfactant 12-2$12 \cdot 2 \mathrm{Br}^{-}$, trimer $12-2-12-2-12 \cdot 3 \mathrm{Br}^{-}$, and tetramer $12-3-12-4-12-3-12 \cdot 4 \mathrm{Br}^{-}$are $0.462 \%$, $0.0675 \%, 0.00898 \%$ and $0.00770 \%$ in mass percentage respectively [5, 9]. 


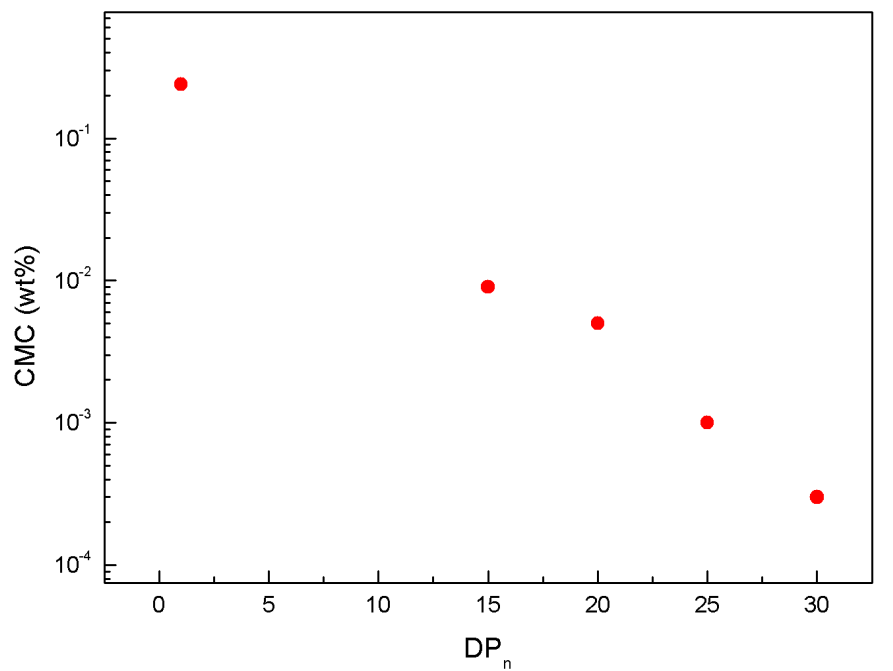

(a)

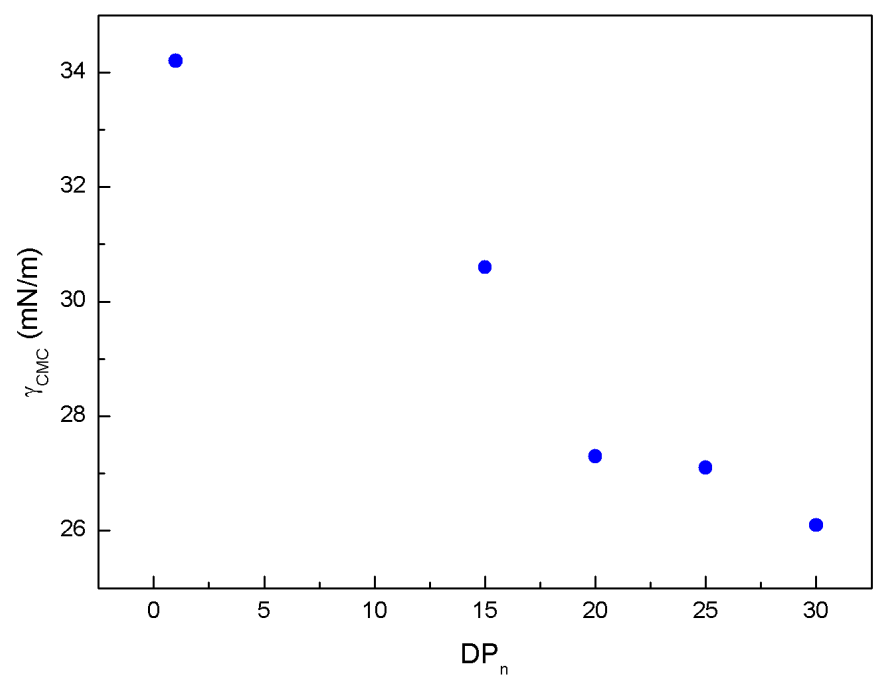

(b)

Fig. 4. (a) CMC and (b) $\gamma_{\mathrm{cmc}}$ of the series of oligomeric alkylpyridinium surfactants and corresponding surfmer

Tab. 3. The CMC and $\gamma_{\mathrm{cmc}}$ of the series of oligomeric alkylpyridinium surfactants and corresponding surfmer

\begin{tabular}{ccc}
\hline Sample & $\begin{array}{c}\text { CMC } \\
(\text { wt\% })\end{array}$ & $\begin{array}{c}\gamma_{\mathrm{cmc}} \\
(\mathrm{mN} / \mathrm{m})\end{array}$ \\
\hline VPB-12 & 0.24 & 34.2 \\
PVPB12-15 & 0.009 & 30.6 \\
PVPB12-20 & 0.005 & 27.3 \\
PVPB12-25 & 0.001 & 27.1 \\
PVPB12-30 & 0.0003 & 26.1 \\
\hline
\end{tabular}


This implies that the oligomeric cationic surfactants synthesized by ATRP have comparable or even better surface activity properties as those obtained from organic synthesis. Furthermore, oligomers PVPB12-n have stronger tendency to micelle than cationic polysoaps which always form intramolecular hydrophobic associates, and decrease only marginally the surface tension which can be only reduced to $50 \mathrm{mN} / \mathrm{m}$, because it was assumed that intramolecular aggregation would result in a complete lack of surface activity [29].

\section{Summary}

In summary, we reported in this work the synthesis and surface activities of a series of 4-vinyl-N-dodecylpyridinium oligomers prepared via ATRP procedure. The polymerization of surfmer 4-vinyl-N-dodecylpyridinium bromide is well controlled in water/IPA mixture at $20{ }^{\circ} \mathrm{C}$ with $\mathrm{Cu}(\mathrm{I})$ complex catalyst. Although curvature of kinetic plots was observed even under these optimized conditions, first-order kinetic plots were obvious and low polydispersity indices were obtained. Furthermore, the observed molecular weight via ${ }^{1} \mathrm{H}$ NMR spectroscopy for the oligomers is consistent with the theoretical one. We believe this represents the first report concerning the direct synthesis of oligomeric cationic surfactants in aqueous solution utilizing ATRP. The physicochemical properties of oligomeric alkylpyridinium surfactants were characterized by investigating surface tension. Their $\gamma_{\mathrm{cmc}}$ and CMC were much lower than those of corresponding surfmer. It was found that increasing polymerization degree could enhance the surface activity properties. The oligomeric surfactants could find potential applications where high surface activity is needed.

\section{Experimental part}

\section{Materials}

4-vinylpyridine, 4-(2-hydroxyethyl) morpholine, triethylamine, $\mathrm{CuBr}, 2$ 2'-bipyridine (bpy) and 2-bromoisobutyryl bromide were all purchased from Aldrich and used as supplied. 1-bromododecane and other reagents including solvents were of commercially available reagent quality. The water used in all experiments was deionized and doubly distilled prior to use. The column chromatography grade silica gel used for removal of the ATRP copper catalyst was purchased from Shenyu Chemical Reagent Co. Ltd. (Chongqing, China) and was filtrated by griddle. Dialysis bag (MW cut-off 3500) was purchased from Guangpu Scientific Instrument Co. Ltd. (Chengdu, China).

\section{Synthesis of Surfmer}

4-Vinyl-N-dodecylpyridinium bromide (VPB12) was prepared by quaternization of 4vinylpyridine with 1-bromododecane. $7.85 \mathrm{~g}(75 \mathrm{mmol})$ of 4-vinylpyridine and $19.94 \mathrm{~g}$ (80 mmol) 1-bromododecane were introduced into a flask and dissolved in $50 \mathrm{ml}$ acetonitrile to form a clear solution. Under magnetic stirring, the reaction was lasted at $60{ }^{\circ} \mathrm{C}$ for $48 \mathrm{~h}$. The reaction was followed by thin-layer chromatography (TLC, silica gel plate) eluted with a mixture of acetone/chloroform $(v / v=6 / 1)$. After the solvent was evaporated off under reduced pressure, surfmer VPB12 was purified by recrystallization from acetone. The final product VPB12 was powder-like and dried under vacuum at $40{ }^{\circ} \mathrm{C}$ for $48 \mathrm{~h}$ prior to being stored in a desiccator. No impurities were detectable by ${ }^{1} \mathrm{H}$ NMR. Yield equals 22.3\%. ${ }^{1} \mathrm{H}$ NMR (Bruker AMX300 300 $\left.\mathrm{MHz}, \mathrm{CDCl}_{3}, \mathrm{TMS}\right)$ for VPB12: $\delta 0.96\left[\mathrm{t}, 3 \mathrm{H}, \mathrm{CH}_{3} \mathrm{CH}_{2}\right], 1.16-1.26[\mathrm{~m}, 22 \mathrm{H}$, 
$\left.\mathrm{CH}_{3}\left(\mathrm{CH}_{2}\right)_{11}\right], 5.18-5.30\left[\mathrm{t}, 2 \mathrm{H}, \mathrm{CH}=\mathrm{CH}_{2}\right.$ ], $5.60\left[\mathrm{t}, \mathrm{H}, \mathbf{C H}=\mathrm{CH}_{2}\right], 9.06\left[\mathrm{t}, 2 \mathrm{H}, \mathrm{N}(\mathbf{C H C H})_{2}\right]$, $8.54\left[\mathrm{t}, 2 \mathrm{H}, \mathrm{N}(\mathrm{CHCH})_{2}\right]$.

\section{Synthesis of Initiator}

2-Morpholinoethyl 2-bromo-2-methylpropanoate (ME-Br initiator) was prepared as previously reported [30], but with revision in this work. In a three-neck flask, $6.55 \mathrm{~g}$ $(0.05 \mathrm{~mol}) 4$ - $(2$-hydroxyethyl) morpholine and $6.06 \mathrm{~g}(0.06 \mathrm{~mol})$ triethylamine were mixed in $60 \mathrm{ml}$ dry toluene for at least $3 \mathrm{~h} .30 \mathrm{ml}$ toluene solution of $11.50 \mathrm{~g}(0.05 \mathrm{~mol})$ 2-bromoisobutyryl bromide was added dropwise to the former toluene solution at 0 ${ }^{\circ} \mathrm{C}$. After addition was completed, the reaction solution was allowed to warm to room temperature, and the final solution was filtered to remove the amine salt. And then the solution was stirred with activated carbon and dried over $\mathrm{MgSO}_{4}$. Finally the toluene solution was filtered and the solvent was removed using the rotary evaporator. Yield equals 85.5\%. ${ }^{1} \mathrm{H}$ NMR (Bruker AMX300 $300 \mathrm{MHz}, \mathrm{CDCl}_{3}, \mathrm{TMS}$ ) for $\mathrm{ME}-\mathrm{Br}$ initiator: 1.95 [s, $\left.6 \mathrm{H}, \mathrm{BrC}\left(\mathrm{CH}_{3}\right)_{2}\right], 2.35-2.64\left[\mathrm{t}, 6 \mathrm{H}, \mathrm{N}\left(\mathrm{CH}_{2}\right)_{3}\right], 3.65[\mathrm{t}, 4 \mathrm{H}$, $\left.\mathrm{O}\left(\mathrm{CH}_{2} \mathrm{CH}_{2}\right)_{2}\right], 4.06\left[\mathrm{t}, 2 \mathrm{H}, \mathrm{O}=\mathrm{CCOCH}_{2}\right]$.

\section{Preparation of Oligomeric Surfactants via ATRP}

A typical protocol for the homo-polymerization of VPB12 via aqueous ATRP using the $\mathrm{ME}-\mathrm{Br}$ initiator to get oligomeric surfactant PVPB12-15 was as follows: $0.28 \mathrm{~g}$ (1 $\mathrm{mmol}) \mathrm{ME}-\mathrm{Br}$ initiator and $6.10 \mathrm{~g}(15 \mathrm{mmol}) \mathrm{VPB} 12$ were co-dissolved in $500 \mathrm{ml}$ water/isopropanol (IPA) mixture $(\mathrm{v} / \mathrm{v}=1 / 2)$. After the reaction mixture was purged with nitrogen for $30 \mathrm{~min}$, the catalyst including $0.14 \mathrm{~g}(1 \mathrm{mmol}) \mathrm{CuBr}$ and $0.32 \mathrm{~g}(2 \mathrm{mmol})$ bpy ligand was added to this stirred solution under nitrogen. The reaction solution was continuously stirred at $20^{\circ} \mathrm{C}$, and immediately became dark brown, indicating the onset of polymerization. After $60 \mathrm{~min}$, an oligomer was obtained at an overall conversion of about $95.0 \%$, as indicated by ${ }^{1} \mathrm{H}$ NMR spectroscopy. The reaction mixture was passed through a silica gel column to remove the ATRP catalyst. After evaporation of the solvent, the product was redissolved in water and dialyzed with a dialysis bag (MW cut-off 3500$)$ with water/IPA mixture $(\mathrm{v} / \mathrm{v}=1 / 2)$ to remove any unreacted surfmer.

The other oligomeric surfactants PVPB12- $n(n=20,25,30)$ were prepared under the identical conditions, and their designed $\mathrm{DP}_{\mathrm{n}}$ was tuned by adjusting concentration ratio of surfmer to $\mathrm{ME}-\mathrm{Br}$ initiator.

\section{Time-Conversion Studies via ${ }^{1}$ H NMR Spectroscopy}

To monitor kinetics of the oligomerization of VPB12 using ME-Br initiator, the mixture solutions to synthesis PVPB12-15 and PVPB12-30 were charged into seven glass tubes and eight respectively. The mixture solutions reacted in the tubes immersed into thermostatic water bath at $20^{\circ} \mathrm{C}$. At timed intervals, the tubes were taken out and oxygen was used to terminate the reaction. The solution mixture was then subjected to ${ }^{1} \mathrm{H}$ NMR analysis directly to determine the kinetics of ATRP using a 300 $\mathrm{M} \mathrm{Hz}$ Bruker spectrometer in $\mathrm{D}_{2} \mathrm{O}$ [30]. By comparing the peak integrals of vinyl signals at $\delta=5.6 \mathrm{ppm}$ and $6.1 \mathrm{ppm}$ to those of the terminal methyl groups in long alkyl chain at $\delta=0.96 \mathrm{ppm}$, the conversion of surfmer during the reaction can be calculated via the formula:

$$
\text { Conversion }=\frac{[M]_{0}-[M]_{t}}{[M]_{0}} \times 100 \%=\frac{I_{0.96} / 3-I_{5.6}}{I_{0.96} / 3} \times 100 \%
$$


where $[\mathrm{M}]_{0}$ means the initial concentration of surfmer, and $[\mathrm{M}]_{\mathrm{t}}$ the current concentration of surfmer during the experiment process. In such a calculation, in order to simplify the situation, initiator was assumed being consumed completely, and chain transfer was negligible; in addition, every polymer chain contained only an initiator end group [23].

\section{Gel Permeation Chromatography (GPC) Analysis}

The GPC analysis was conducted using a Water 515 liquid chromatograph instrument connected with a Waters 2410 refractive index detector. The gel permeation column was OHpak KB-803 $(7.8 \times 300 \mathrm{~mm})$, and the solvent used was distilled water. PEG was used as polymer standard.

\section{Measurement of Static Surface Tension}

Surface tension of the oligomeric surfactants and surfmer was measured with Wilhelmy plate technique (surface tensiometer BZY-1, Shanghai Hengping Instrument, China) as described previously [31]. Measurements were taken at $25 \pm$ $0.5{ }^{\circ} \mathrm{C}$ until constant surface tension values was reached. The $\mathrm{CMC}$ values were taken at the intersection of the linear portions of the plots of the surface tension against the logarithm of the surfactant concentration. Solution confected with a sort of product was diluted continuously with doubly distilled water, while the concentration of the solution was reduced as half as former one each time. The surface tension of the solution was measured three times at each concentration, and then the average of the three values was gained.

\section{Acknowledgements}

The financial support from Sichuan Provincial Sichuan Provincial Bureau of Science and Technology (2008GZ0004, 2010JQ0029) for this work is greatly acknowledged.

\section{References}

[1] Zana, R.; Xia, J. (Eds.) Gemini Surfactants: Synthesis, Interfacial and SolutionPhase Behavior, and Applications. Dekker: New York, 2003.

[2] Danino, D.; Talmon, Y.; Levy, H. et al. Science 1995, 269, 1420.

[3] Zana, R.; Levy, H.; Papoutsi, D. et al. Langmuir 1995, 11, 3694.

[4] Esumi, K.; Taguma, K.; Koide, Y. Langmuir 1996, 12, 4039.

[5] Yoshimura, T.; Yoshida, H.; Ohno, A. et al. J. Colloid Interface Sci. 2003, 267, 167.

[6] Laschewsky, A.; Wattebled, L.; Arotcarena, M. et al. Langmuir 2005, 21, 7170.

[7] Hou, Y.; Cao, M.; Deng, M. et al. Langmuir 2008, 24, 10572.

[8] Kim, T.; Kida, T.; Nakatsuji, Y. et al. Langmuir 1996, 12, 6304.

[9] In, M.; Bec, V.; Aguerre-Chariol, O. et al. Langmuir 2000, 16, 141.

[10] Zana, R. Adv. Colloid Interface Sci. 2002, 97, 205.

[11] Zana, R.; Talmon, Y. Nature 1993, 362, 228.

[12] Menger, F. M.; Littau, C. A. J. Am. Chem. Soc. 1991, 113, 1451.

[13] Menger, F. M.; Keiper, J. S. Angew. Chem. Int. Ed. 2000, 39, 1906.

[14] Wattebled, L.; Laschewsky, A.; Moussa, A. et al. Langmuir 2006, 22, 2551.

[15] Summers, M.; Eastoe, J. Adv. Colloid Interface Sci. 2003, 100-103, 137.

[16] Guyot, A. Adv. Colloid Interface Sci. 2004, 108-109, 3.

[17] Ong, C. L.; Gan, L. M.; Ong, C. K. et al. J. Phys. Chem. B 1999, 103, 7573. 
[18] Salamone, J. C.; Snider, B.; Fitch, W. L. Macromolecules 1970, 3, 707.

[19] Dreja, M.; Pyckhout-Hintzen, W.; Tieke, B. Macromolecules 1998, 31, 272.

[20] Pyrasch, M.; Tieke, B. Colloid Polym. Sci. 2000, 278, 375.

[21] Wang, J. S.; Matyjaszewski, K. J. Am. Chem. Soc. 1995, 117, 5614.

[22] Patten, T. E.; Xia, J.; Abernathy, T. et al. Science 1996, 272, 866.

[23] Li, Y. T.; Armes, S. P.; Jin, X. P. et al. Macromolecules 2003, 36, 8268.

[24] Weaver, J. V. M.; Bannister, I.; Robinson, K. L. et al. Macromolecules 2004, 37, 2395.

[25] Xin, Y.; Hu, Y.; Zeldin, M. et al. Macromolecules 1993, 26, 4670.

[26] Tsarevsky, N. V.; Pintauer, T.; Matyjaszewski, K. Macromolecules 2004, 37, 9768.

[27] Mitsukami, Y.; Donovan, M. S.; Lowe, A. B. et al. Macromolecules 2001, 34, 2248.

[28] Pintauer, T.; Matyjaszewski, K. Chem. Soc. Rev. 2008, 37, 1087.

[29] Laschewsky, A. Adv. Polym. Sci. 1995, 124, 1.

[30] Robinson, K. L.; Weaver, J. V. M.; Armes, S. P. J. Mater. Chem. 2002, 12, 890.

[31] Sun, Y.; Feng, Y.; Dong, H. et al. Surf. Sci. 2007, 601, 1988. 